\title{
INSTRUMENTOS PARA LA OPERACIÓN DE LA CATARATA (1790)
}

\author{
BARBÓN JJ ${ }^{1}$, ÁLVAREZ-SUÁREZ ML ${ }^{2}$
}

El conocimiento de las prácticas y usos de la primitiva oftalmología nos permite valorar no sólo los enormes progresos conseguidos, sino también recordar el triste futuro al que estaban avocados los pacientes en la mayor parte de las enfermedades oculares. El presente artículo pretende lanzar una mirada sobre el estado de la cirugía de cataratas en un tiempo de transición, a finales del s. XVIII, en que se iba imponiendo la cirugía extracapsular ideada por Daviel en 1745, pero en el que aún seguía vigente la reclinación del cristalino. Para ello vamos a utilizar como fuente el Tratado de las enfermedades de los ojos y de los medios y operaciones propias para su curación del francés Louis Florent Deshais-Gendron, sirviéndonos de las palabras del propio autor en el relato. El libro en castellano se edita en Madrid en 1790, en 2 volúmenes, traducido directamente de la edición francesa (Traité des maladies des yeux \& des moyens \& des opérations propres à leur guérison, 1770), por el Dr. Francisco Marín, cirujano oculista de los Reales Hospitales General y Pasión de la Corte. Deshais-Gendron fue el primer profesor oficial de oftalmología en Francia, en 1765, cuando se fundó en París una Cátedra de Oftalmología impulsada por Lamartinière, primer cirujano del rey, al que dedica el libro. En la página de presentación se intitula como Profesor y Demostrador Real de las Enfermedades de los ojos en las Escuelas de Cirugía de París y acompaña cada tomo de una lámina, la segunda con los instrumentos necesarios para la operación de catarata.

En el segundo tomo, en el extenso capítulo XXII (49 páginas), titulado De las enfermedades del cristalino y particularmente de la catarata, DeshaisGendron nos explica con detalle los dos modos de intervención vigentes, el abatimiento o depresión y la extracción. El autor hace las siguientes consideraciones sobre la preparación del enfermo, el lugar y las condiciones, similares en ambos métodos: «Se preparará con un régimen conveniente algún tiempo antes de la operación, apartando al enfermo de los alimentos groseros, ordenando algunas lavativas, sangrándole una ó dos veces según la plenitud, purgándole suavemente, esto es, sin irritarle, para que los humores no se alteren, lo que se repetirá por poca apariencia de cacochîmia [malicia de los humores] que tenga.» «...se escogerá un día claro, y que no halla apariencia de lluvia... Se cuidará

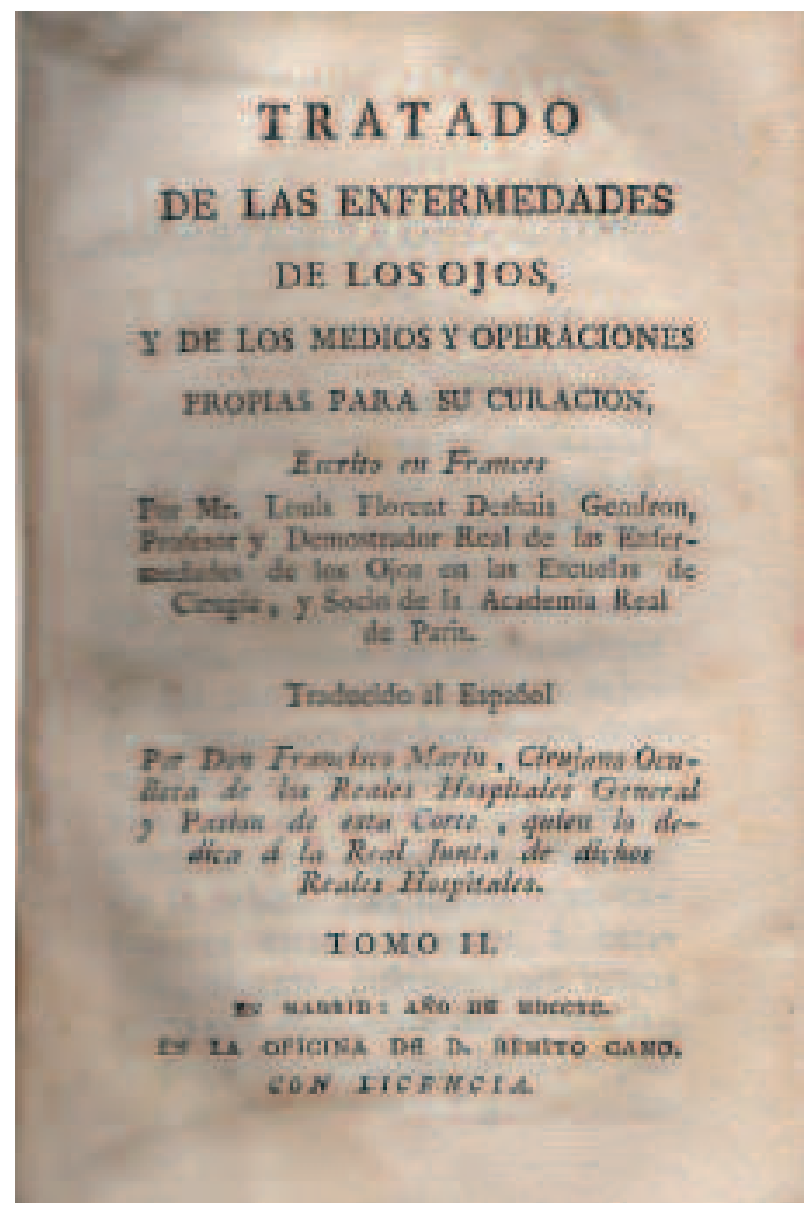

\footnotetext{
${ }^{1}$ Licenciado en Oftalmología. Hospital San Agustín de Avilés. Asturias. España. E-mail: jjbarbon@telecable.es

2 Doctor en Oftalmología. Hospital Álvarez-Buylla de Mieres. Asturias. España.
} 
antes de la operación de que el enfermo tome algún alimento para que no cayga en desmayo... La hora más cómoda es de las diez á las doce, eligiendo para ello un sitio bien claro, pero que no dé el sol. Se tapará el otro ojo, si ve con él, con una compre$s a$, sostenida con un vendaje puesto obliqüamente.»
La depresión del cristalino (fig. 13) se realizaba con agujas bien afiladas, sin anestesia ni ninguna medida antiséptica y tras explicar detalladamente la posición de cirujano y enfermo, así como la manera de realizar la apertura palpebral, Deshais-Gendron expone la técnica que consistía en introducir

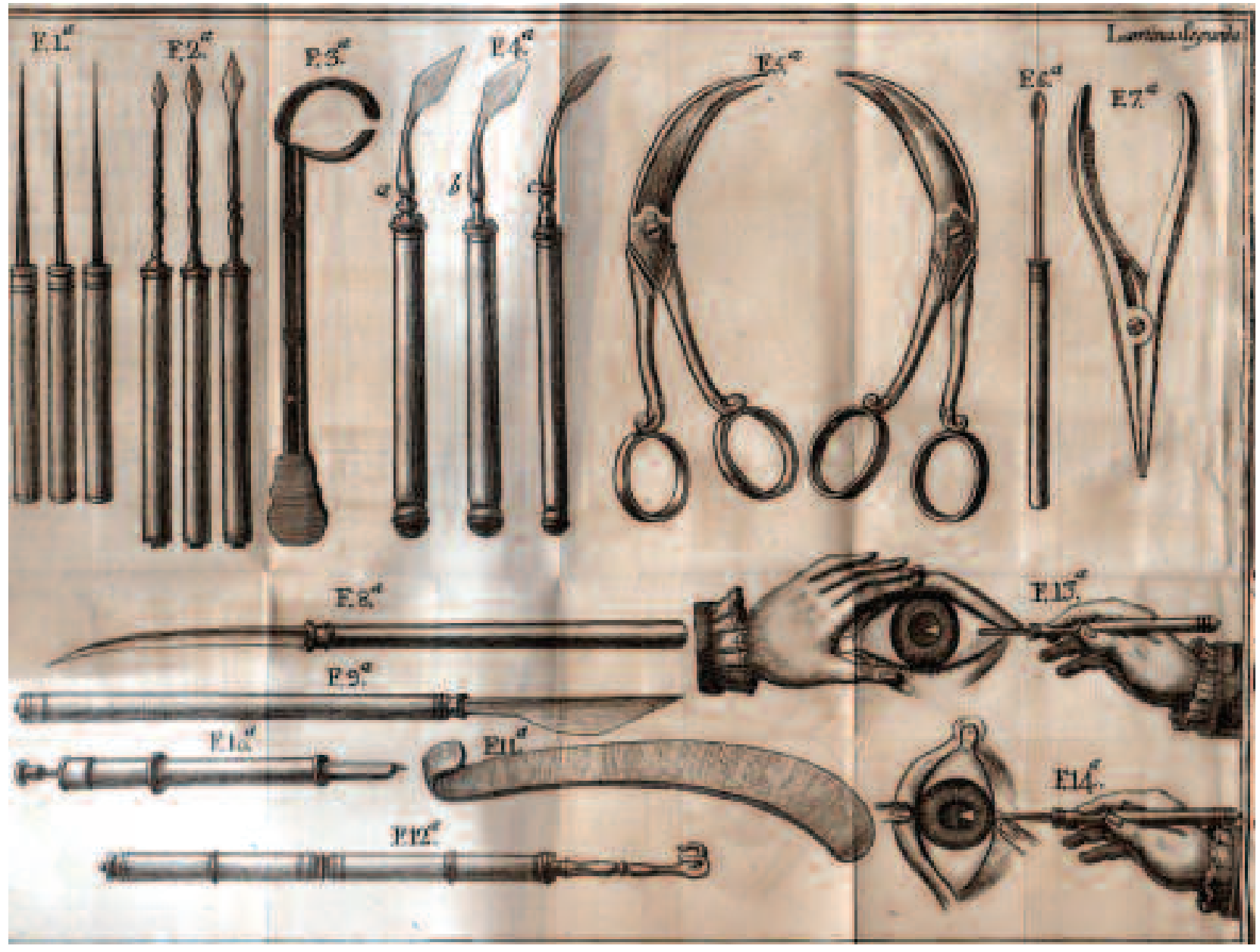

Instrumentos para la operación de la catarata (1790):

Fig. 1.- Tres agujas redondas que antiguamente se usaban para la depresión de la catarata.

Fig. 2.- Otras 3 planas, de figura de lengua de víbora, que son las que hoy día se usan.

Fig. 3.- Speculum Oculi, que los antiguos se servían de él para sujetar los párpados en la depresión de la catarata.

Fig. 4.- Tres agujas o lanzas, corvas, puntiagudas y cortantes, de M. Daviel para hacer la extracción de la catarata.

Fig. 5.- Dos pares de tijeras para dilatar la incisión de la córnea.

Fig. 6.- Cucharita de oro o plata para levantar la porción de la córnea cortada.

Fig. 7.- Pinzas para sacar algunas porciones de la cápsula del cristalino o del mismo cristalino.

Fig. 8.- Bisturí o cuchillo de M la Faye para hacer la incisión de la córnea.

Fig. 9.- Otro bisturí de M. Poyet más ancho en su hoja para hacer el corte de la córnea.

Fig. 10.- Kistotomo de M. la Faye para dilatar y picar la cápsula del cristalino.

Fig. 11.- Garfio de M. Berenguer para levantar el párpado superior.

Fig. 12.- Herina para sujetar el globo en la parte inferior.

Fig. 13.- Un ojo que demuestra como se hace la operación de la catarata por depresión o abatimiento.

Fig. 14.- Otro que señala el modo de operar por extracción, de M. Daviel. 
«... la aguja por lo blanco del ojo, a dos líneas poco más o ménos del borde de la córnea... de este modo se hallará la aguja detrás del cristalino que impide verla... se conducirá su punta á la parte superior y anterior del cristalino, lo que se hará baxando un poco la mano... para apoyar con la aguja sobre la catarata, que por estos movimientos se abate; se reiteran hasta desaloxar al cristalino de su cavidad ó engaste en el vítreo: ganada la parte superior de la catarata, se apoyará con el plano de la aguja sobre ella, para colocarla debaxo de la pupila en la parte inferior del vítreo».

La extracción (fig. 14), con similar preparación y con el enfermo sentado se iniciaba introduciendo la primera aguja «... por la parte inferior de la córnea en la cámara anterior inmediato á la esclerótica, y la guiará hasta lo alto de la pupila, evitando herir el iris, y después la sacará suavemente, ladeándose un poco hacia el pequeño ángulo, a fin de agrandar su incisión para introducir la aguja obtusa, con la qual dilatará el corte comenzado... para abrir la córnea en forma de media luna según su redondez... tomara el Cirujano las tixeras corvas convexâs... y acabará la sección de un lado y de otro un poco más arriba de la pupila.» «Tomará después la pequeña spátula, con la qual levantará suavemente la parte de la cornea separada, y cortará con la pequeña aguja puntiaguda y cortante la parte anterior de la membrana cristalina... estando bien cortada, se puede sacar con las pequeñas pinzas. Cortada la membrana cristalina, se comprimirá suavemente el globo en su parte superior con el pulgar de la mano derecha... por este medio la compresión sobre el cristalino es más exâcta, y el cuerpo vítreo entónces tomando una figura oblonga obliga al cristalino a salir de su engarce, si no ha contraído alguna adherencia con la cara posterior del iris. Se puede para evitar la ruptura de la membrana posterior del cristalino, e impedir la salida de humor vítreo... dando al enfermo una situación horizontal durante la operación, en lugar de la perpendicular que se da ordinariamente, esto es, haciéndole echar ácia atras.» Que en esto el temor de la rotura de la cápsula posterior, entonces cuita, sigue siendo el mismo. «Este modo de operar la catarata por la extracción se debe a M. Daviel que nos ha dado los instrumentos que acabamos de exponer para hacerla.» También explica el manejo de algunos instrumentos mejorados que facilitan el procedimiento como los bisturís para la incisión corneal de de la Faye y de Poyet, y un quistotomo diseñado por de la Faye.

Finalmente hace unas consideraciones comparando las complicaciones de ambas técnicas quirúrgicas. «En la depresión se presentan muchos más accidentes que en la extracción, porque en la depresión se hace todo en cámara posterior, que es el lugar de partes más respetables, y así los accidentes que pueden ocurrir son la picadura de los nervios del círculo y ligamento ciliar, la de los procesos ciliares, la ofensa de los vasos de la chôroydes, la separación de la retina, la ruptura del cristalino en muchas partes, su substancia disuelta y repartida en las cámaras, el rompimiento de las cápsulas y celdillas del cuerpo vítreo: en lugar que en la extracción se opera en la cámara anterior, y los accidentes que pueden sobrevenir no son en mucho tan considerables como los que acontecen en el antigua método».

«Los que pueden sobrevenir en la extracción son la herida del iris, las violencias que la pupila sufre al dar paso al cristalino, la pérdida de humor vítreo... y en fin aquellos que puedan venir después de la operacion, como inflamaciones, dolores, supuración y vertimiento de todo el globo.» (Ya tenían expulsivas).

«Si no aconteciese cosa alguna, y que el ojo estuviese sin inflamación ni dolor, se le permitirá comer al enfermo una sopa clara á los quatro ó cinco días, y á los otros quatro ó cinco se le permitirá un poco de vianda... Si á los nueve ó diez días no hubiese venido ningún accidente, se le taparán los ojos con un tafetán negro, pendiente de una venda circular por la frente, y suelto por debaxo, á fin de acostumbrar los ojos á la luz, la que se hará entrar en al aposento por grados.»

\section{BIBLIOGRAFÍA}

- Deshais-Gendron LF. Tratado de las enfermedades de los ojos y de los medios y operaciones propias para su curación. Madrid: 1790; 189-237.

- Munoa JL, Cotallo JL. El nacimiento de la especialidad oftalmológica. In: Cotallo de Cáceres JL, Hernández. Benito E, Munoa Ruiz JL, Leoz de la Fuente G. Historia de la Oftalmología Española. Madrid: LXIX Ponencia de la Sociedad Española de Oftalmología: 1993; 171-180.

- Álvarez Suárez. M. Instrumentos necesarios para la operación de la catarata senil (1904). Arch Soc Esp Oftalmol 2001; 76: 447-448. 